\title{
Positive Effect of Oral Supplementation with Glycosaminoglycans and Antioxidants on the Regeneration of Osteochondral Defects in the Knee Joint
}

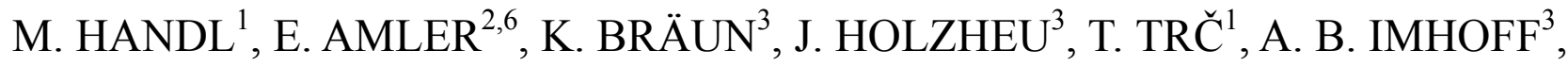 \\ A. LYTVYNETS ${ }^{4}$, E. FILOVÁ ${ }^{2,6}$, H. KOLÁŘOVÁ ${ }^{5}$, A. KOTYK ${ }^{4}$.V. MARTÍNEK ${ }^{3}$ \\ ${ }^{1}$ Orthopedic Clinic, Charles University, Prague, Czech Republic, ${ }^{2}$ Institute of Experimental \\ Medicine, Academy of Sciences of the Czech Republic, Prague, Czech Republic, ${ }^{3}$ Department of \\ Orthopedic Sports Medicine, Technical University Munich, Germany, ${ }^{4}$ Institute of Physiology, \\ Academy of Sciences of the Czech Republic, Prague, Czech Republic, ${ }^{5}$ Institute of Medical \\ Biophysics, Medical Faculty, Palacký University in Olomouc, Czech Republic and ${ }^{6}$ Institute of \\ Biophysics, Second Faculty of Medicine, Charles University in Prague, Czech Republic
}

Received November 29, 2005

Accepted February 27, 2006

On-line available March 23, 2006

\begin{abstract}
Summary
The effect of oral supplementation with glycosaminoglycans (GAG) and radical scavengers (vitamin E/selenium) on the regeneration of osteochondral defects was investigated in rabbits. After introduction of defined osteochondral defects in the knee joint, groups of ten animals were given a GAG/vitamin E/selenium mixture or a placebo (milk sugar) for 6 weeks. Following sacrifice, histological and histochemical analysis was performed. The amount of synovial fluid was increased in the placebo group, while the viscosity of the synovial fluid was significantly enhanced in the GAG group. The amount of sulfated GAG in the osteochondral regenerates $(8.8 \pm 3.6 \%$ vs. $6.0 \pm 5.6 \% ; p<0.03)$ was significantly higher in the GAG group. In both groups, the GAG amount in the cartilage of the operated knee was significantly higher than in the non-involved knee $(p<0.05)$. Histological analysis of the regenerates in the GAG group was superior in comparison with the placebo group. For the first time, a biological effect following oral supplementation with GAG was demonstrated in healing of osteochondral defects in vivo. These findings support the known positive clinical results.
\end{abstract}

Key words

Cartilage • Glycosaminoglycans • Oral supplementation • Osteochondral lesion

\section{Introduction}

Typically, damage to the joint cartilage in major joints usually leads to osteoarthritis (OA) and thus causes higher health costs as well as employment and salary limitations for a given socio-economic group of the society (MacLean et al. 1998, Steinmeyer 2000). While older patients with osteoarthritis profit from joint 
replacements, treatment of younger patients remains uncertain due to the limited life of the prosthesis (Sharkley et al. 2002). Despite the worldwide scientific effort the possibilities of treatment of cartilage damage that have been presented so far have still not been optimized (Martinek and Imhoff 2003).

Cartilage lesions are most often treated in a conservative manner (Erggelet et al. 2000, Steinmeyer 2000, Towheed 2002). Very often, only the antiinflammatory effect of drugs is employed, but the basic causes of joint pathology are not decisively solved by this approach (Steinmeyer 2001). During the last 10 to 15 years, the basics of peroral medical therapy have been enriched by new aspects of OA research (Erggelet et al. 2000, Martinek and Imhoff 2003). Aside from NSAID, various other substances have been found to display a potential chondroprotective effect in the treatment of osteoarthritis (Debi et al. 2000, McAlindon et al. 2000ab, Reginster et al. 2001, Brief et al. 2001, Raynauld et al. 2002, Towheed 2002).

Various agents, including GAG (chondroitin sulfate, D-glucosamine sulfate, hyaluronic acid), piroxicam, ademetionin, oxaceprol and heparinoids (Steinmeyer 2000) have been shown to exert not only an anabolic effect on chondrocytes but also an inhibitory function on free catabolic metabolites under in vitro and in vivo conditions (Harmand et al. 1987, Bassleer et al. 1992, Vivien et al. 1993, Homandberg et al. 1997, Ronca et al. 1998, Uebelhart et al. 1998, Towheed 2002). However, until now, it has not been possible to directly prove a causal link between orally applied agents and the structural changes within the hyaline cartilage or within cartilage regenerates (Steinmeyer 2000, Brief et al. 2001). In spite of being supported by positive clinical results, these substances are discussed controversially and are not accepted as OA-modifying drugs (Altman and Moskowitz 2002, Felson and Anderson 2002).

GAG, whose positive effect on chondrocyte metabolism is well known, have meanwhile become prevalent in the peroral treatment of cartilage pathologies (Vidal et al. 1978). In recent controlled, randomized double-blind prospective studies, significant reduction of symptoms and positive radiological changes in knee joints have been demonstrated in patients with osteoarthritis following long-term GAG supplementation (Reginster et al. 2001, Pavelka et al. 2002). However, there has been no breakthrough in osteoarthritis therapy as yet. The question remains whether the peroral intake of substances such as GAG can be effective in location of the pathology or whether they are destroyed during the digestion process.

The present animal study investigates the effect of perorally applied chondrogenic agents in the regeneration of osteochondral defects. For the evaluation, a designed preparation containing the mixture of GAG in their natural composition - as well as vitamin $\mathrm{E}$ and selenium acid radical scavengers, were used to prove the biological effect in the damaged joints.

\section{Methods}

\section{Surgery}

For the experiments, 20 adult female gray Chinchilla rabbits with an average weight of $2.5 \pm 0.3 \mathrm{~kg}$ were used and randomized into two groups. The knee joints of rabbit and man are anatomically comparable; in addition rabbits have already been used as an experimental animal for cartilage research in the knee joint (Martinek et al. 2002).

The procedure was performed unilaterally. Under mask anesthesia, anteromedial knee arthrotomy was performed and the patella was moved laterally. In $90^{\circ}$ knee flexion, a $3.5 \mathrm{~mm} \times 3.5 \mathrm{~mm}$ (width $\times$ depth) osteochondral defect was created in the trochlea femoris (Fig. 1). After thorough lavage and cleansing from residual blood the knee joint was closed and sutured in two layers.

\section{Postoperative treatment and drug distribution}

Postoperatively the animals were not immobilized and they were allowed to move freely in their cages. Prophylactic peroral antibiotic therapy with sulfonamide was conducted during the first three postoperative days. The animals were weighed once a week. Both groups received the same food but those within the GAG study group were additionally fed $50 \mathrm{mg} / \mathrm{kg}$ of the GAG-mixture with $10 \mu \mathrm{g}$ selenium and $5 \mathrm{mg}(10 \mathrm{mg} / \mathrm{kg})$ vitamin E (KnorpelPlus ${ }^{\circledR}$; Nutritect, Germany) in tablet form. The animals in the control group were given placebo tablets (milk sugar $50 \mathrm{mg} / \mathrm{kg}$ ). Six weeks after the operation all animals were sacrificed. The amount and viscosity of the synovial fluid were measured and the cartilage of both knee joints macroscopically analyzed.

\section{Sample collection and analysis}

The exposed joints were photographed and biopsies $(\approx 10 \mathrm{mg}$ ) were sampled from the tissue filled 


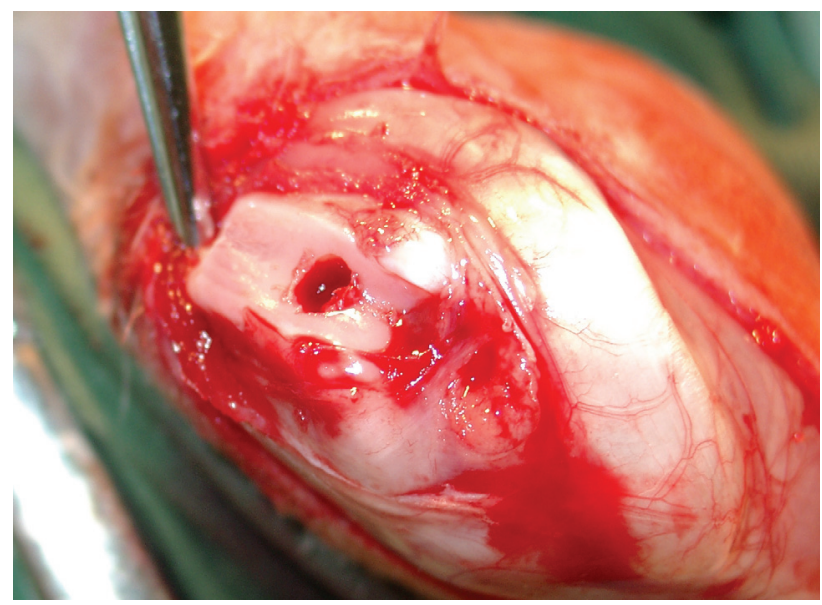

Fig. 1. Operation site: creation of the osteochondral defect in the trochlea femoris in the rabbit knee joint.

inside the osteochondral defects (Fig. 2), from the surrounding cartilage and from the cartilage of the contralateral knee joint. The biopsies were analyzed for their total sulfated content as well as for the amount of N/O-sulfated GAG. The total content of sulfated GAG was determined fluorometrically (see below). The tissue was washed, lyophilized in a vacuum desiccator and the dry weight was dermined. The samples were then enzymatically split into $1 \mathrm{ml}$ of a $0.5 \%$ papain-buffer solution in a $65^{\circ} \mathrm{C}$ bath and 1,9-dimethylmethylene blue (DMB) was added to the aliquot. Finally, absorption was measured spectrophotometrically at $535 \mathrm{~nm}$ (Farndale et al. 1986). The GAG amount was determined by extrapolation from the standard curve with shark chondroitin sulfate (Sigma, Germany). The defects with the surrounding cartilage were removed, denatured in formaldehyde, decalcified in EDTA, cut into $5 \mu \mathrm{m}$ layers, stained by hematoxylin/eosin (H/E) and histologically analyzed with regard to the type, orientation and number of cells as well as the character of the matrix.

\section{Fluorescence measurements}

Due to the very small volume, the viscosity of the synovial fluid was measured using a fluorescence polarization of FITC-marked polylysine as the fluorescence probe using Perrin equation for viscosity determination as described by Amler et al. (1990).

Non-enzymatic glycation has been estimated from the fluorescence intensity of natural fluorescent products. Steady-state excitation (emission $440 \mathrm{~nm}$ ) and emission (excitation $335 \mathrm{~nm}$ or $370 \mathrm{~nm}$ ) spectra were recorded on Perkin-Elmer LS50B spectrofluorometer. For determination of the AGEs formation dependence on the age, a $600-\mu 1$ sample of the incubation solution was
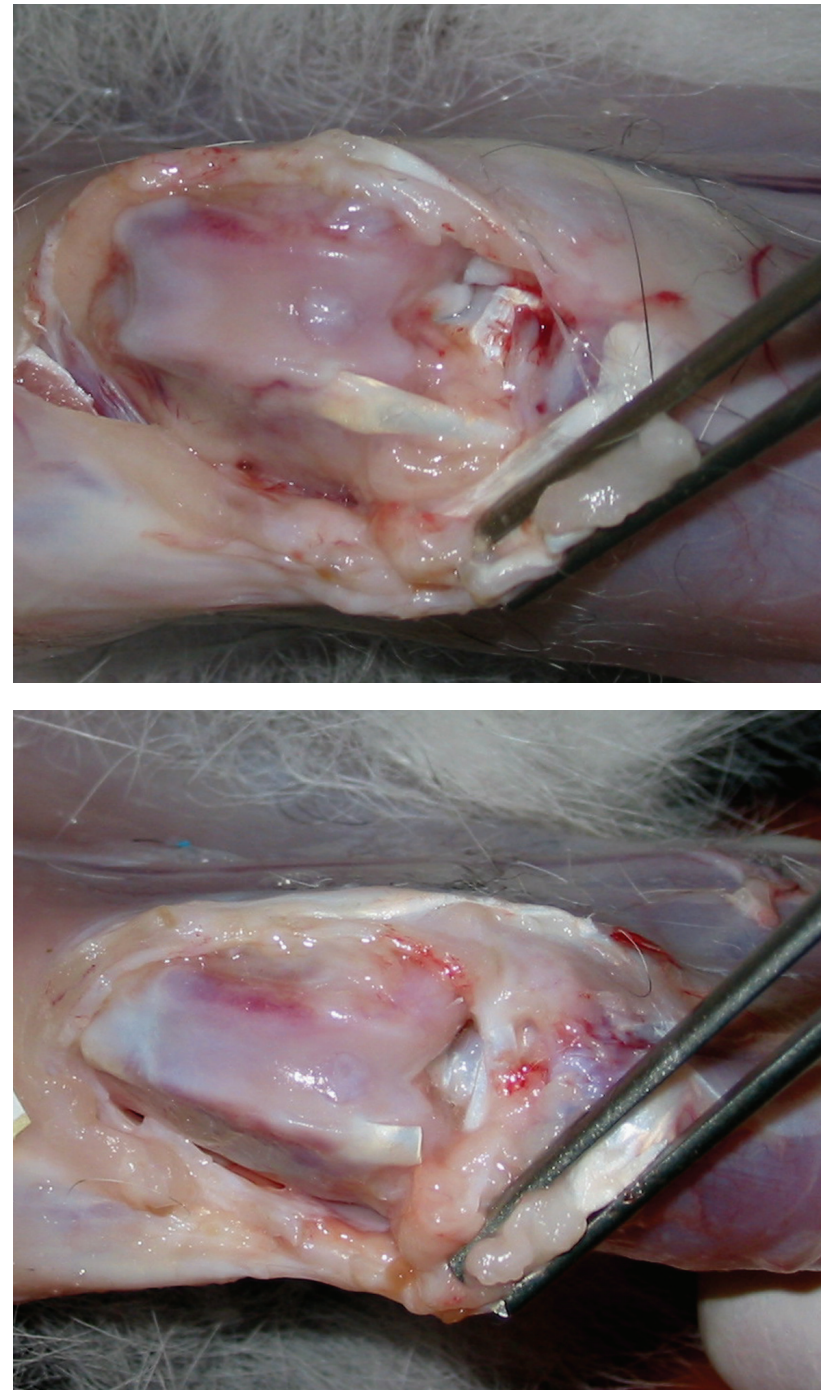

Fig. 2. The osteochondral defect after 6 weeks filled with fibrocartilage tissue placebo (upper), GAG group (lower).

diluted in $2 \mathrm{ml}$ of $20 \mathrm{mM}$ Tris- $\mathrm{HCl}(\mathrm{pH} \mathrm{7.4)}$ and the fluorescence intensities of the solution at excitation/emission wavelengths $335 / 400 \mathrm{~nm}$ and $370 / 440 \mathrm{~nm}$ were recorded at $37{ }^{\circ} \mathrm{C}$. Both excitation and emission band passes were set to $5 \mathrm{~nm}$, the signal was integrated for $5 \mathrm{~s}$.

The fluorescence of pentosidine and nonspecific glycation was related to the fluorescence of proteins.

\section{Statistics}

The values are shown as the mean \pm S.E.M. The data were analyzed statistically by paired Student's t-test. Statistical significance was set at $p<0.05$.

\section{Results and Discussion}

The postoperative course developed without complications in all animals. With regard to the behavior 


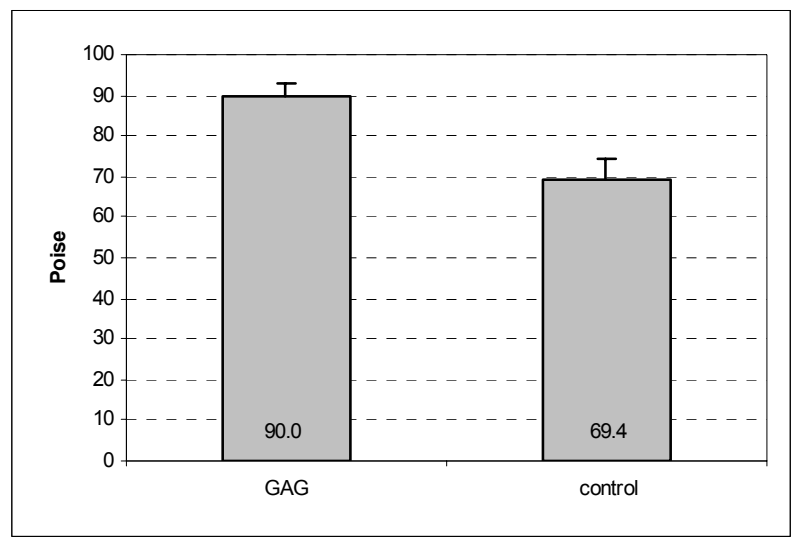

Fig. 3. Viscosity of the synovial fluid $(p<0.001)$.

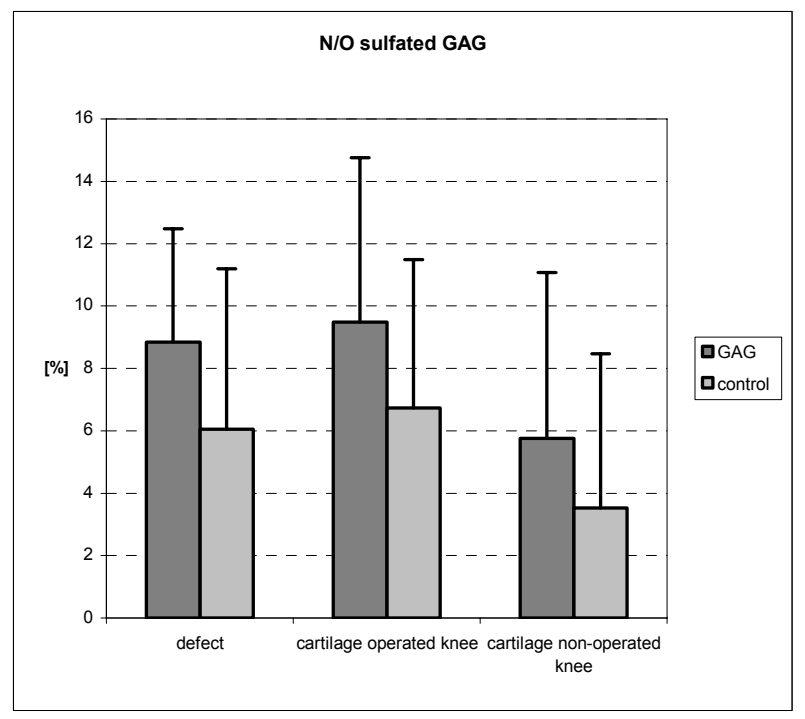

Fig. 4. The amount of sulfated GAG as share of the total weight of the regenerated tissue in the osteochondral lesions.

and mobility of the rabbits, it was not possible to discern any difference between the two groups. All animals were able to run and jump on all extremities within the whole period after surgery. In the GAG group a significantly higher ( $p<0.0004)$ weight increase $(1.78 \pm 0.19 \mathrm{~kg})$ was detected in comparison with the control group $(1.13 \pm$ $0.37 \mathrm{~kg})$. The amount of synovial fluid in the control group $(0.18 \pm 0.22 \mathrm{ml})$ was significantly higher $(\mathrm{p}<0.02)$ than in the GAG group $(0.01 \pm 0.01 \mathrm{ml})$, while the viscosity of the synovial fluid was significantly higher in the GAG group $(9.0 \pm 0.29$ pascal $\cdot$ second vs. $6.94 \pm 0.49$ pascal-second; p <0.001) (Fig. 3). Comparing the GAG group with the placebo group, the total amount of GAG content was $12.8 \pm 3.6 \%$ vs. $9.6 \pm 7.8 \%$; $(\mathrm{p}<0.16)$ in the osteochondral defects, $13.8 \pm 6.0 \%$ vs. $9.9 \pm 5.1 \%$; $(p<0.07)$ in the surrounding cartilage and $8.9 \pm 6.3 \%$ vs.
$5.9 \pm 5.1 \% ;(p<0.15)$ in the cartilage of the contralateral knee joint. The amount of the sulfated GAG in the osteochondral defects of the GAG group $(8.8 \pm 3.6 \%)$ was significantly higher $(\mathrm{p}<0.03)$ than in the control group $(6.0 \pm 5.6 \%)$ (Fig. 4). In both groups the total GAG content in the cartilage of the operated knee joint was also significantly higher $(p<0.05)$ than in the nonoperated knee joint.

Histological analysis showed a higher organization level of the cartilage-like repair tissue in the healed osteochondral defect in the GAG group in comparison with the placebo group.

Proteoglycans are complex macromolecules composed of numerous GAG and oligosaccharide chains which are located in the external space of the cartilage collagen network (Buckwalter and Mankin 1998). They bind water and positive ions in the cartilage substance and, in addition, form a viscous elastic layer, which lubricates and protects the cartilage surface (Brief et al. 2001). The presence of these aggregates is the most important factor for the biomechanical properties of the hyaline cartilage in the joints. The GAG, which are mainly composed of keratan sulfate, dermatan sulfate, heparan sulfate, chondroitin sulfate and hyaluronic acid, form the most important part of the proteoglycans (Buckwalter and Mankin 1998).

The loss of proteoglycans in the process of cartilage degeneration and osteoarthritis due to the imbalance in the exchange of matrix proteins, has a significant negative consequence on the biomechanical properties of the cartilage (O'Driscoll 1998). Theoretically, the exogenous intake of GAG can enhance the synthesis of proteoglycans in the cartilage and compensate for the loss of the matrix. This potential therapeutic effect has already been shown in clinical studies which report the improvement of clinical symptoms and radiological parameters in patients with cartilage damage or osteoarthritis by peroral doses of GAG (Reginster et al. 2001).

Experiments in vitro confirm the effect of GAG on chondrocytes (Vidal et al. 1978, Bassleer et al. 1992, Piperno et al. 2000). In animal studies, substances like chondroitin sulfate or glycosamine sulfate not only cause an increase in the growth rate and production of proteoglycans, but also specifically interrupt catabolic processes such as the collagenolytic activity of chondrocytes or support an antiinflammatory function in the joints (Pipitone 1991, Setnikar et al. 1991, Piperno et al. 2000). 
Until now, a direct proof demonstrating structural changes in the cartilage following peroral uptake of GAG has been lacking in vivo (Steinmeyer 2000). Despite numerous experiments, the biological availability of peroral GAG remains uncertain (Brief et al. 2001). Increased levels of the hyaluronic acid and sulfatized GAG in the plasma and synovial fluid can be obtained and proved in humans after intake of exogenous doses of GAG. However, the question of the required GAG concentration and the relevance of these molecules for chondropathy still remains open (Steinmeyer 2000).

In the present study it was possible to demonstrate that a continuous peroral supply of GAG mixture leads to structural changes in the regenerated cartilage in the knee joints in rabbits. Six weeks after induction of an osteochondral defect in the knee joint, an increased enrichment of sulfated GAG in the newly formed fibrocartilage was found as a result of peroral substitution with GAG. Furthermore, an increased level of total GAG was measured in the surrounding cartilage of the treated knee joint. These findings suggest that the cartilage tissue is supplied not only by blood vessels which are responsible for the increase of GAG in the subchondral regenerate of the fibrocartilage - but also by the synovial fluid. In addition, the significantly increased viscosity of the synovial fluid could be demonstrated as a consequence of the oral GAG supplementation.

The total GAG amount in the surrounding cartilage was significantly increased in both groups and comparable between the GAG and the control group. This finding supports the hypothesis that the performed surgical intervention led to the activation of chondrocytes and consequently to an anabolic activity in the joint. According to Lotz (2001), cytokines released by the acute surdical injury were responsible for triggering this effect.

Besides these changes in the cartilage composition, the significant reduction of joint effusion in the treated joint was measured in the GAG group. However, this finding could be a function of all the KnorpelPlus-supplied agents and cannot be assigned specifically to GAG, vitamin E or selenium by this study. According to previous experiments, GAG possess an antiinflammatory effect and are able to reduce synovial inflammation (Setnikar et al. 1991, Ronca et al. 1998). In clinical studies, formation of effusions in patients is significantly diminished following peroral supplementation with GAG or chondroitin sulfate (Noack et al. 1994, Debi et al. 2000, McAlindon et al. 2000a).

Vitamin E is commonly known as an oxygen radical scavenger and it has therefore been clinically used for years in various pathologies as an antioxidant agent (Grimble 1994). Selenium, an important trace element in food, is also considered to have antioxidative functions (Combs 2001). Peng and Yang (1991) described the role of selenium for the Kaschin-Beck disease, which is an endemic disease in China, with symptoms of both osteoarthritis and generalized cartilage destruction. This syndrome can be caused either by a deficit of selenium or by high levels of free radicals in the ground water (Peng et al. 1992). Peng and Yang (1991) were able to show that selenium significantly lowers the production of lipid peroxidase and thus serves as a protective factor for this disease. According to their study, reduction of the effusion in the treated joints in our study can be taken as a function of these oxygen radical scavengers, neutralizing aggressive cytokines.

Interestingly, the body weight of animals in the GAG-substituted group was significantly higher than that of the animals in the placebo group. This effect could be explained by an increased water content in the soft tissues and the cartilage following increased concentration of GAG. However, this hypothesis is not supported by data available in the literature since such a phenomenon has not been described before. A possible, hypothetical, explanation is the well-being with pain reduction in animals of the GAG group that leads to a higher weight increase.

The used preparation of GAG represents an important aspect in our study. The KnorpelPlus preparation contained a whole spectrum of substances that are incorporated in normal hyaline cartilage tissue and thus differed significantly from the so-called monopreparations that are composed only of a single GAG. In our opinion, the used preparation is compatible with the hypothesis that as many building stones as possible are necessary for reconstruction of the connective tissue such as hyaline cartilage. Glycosaminoglycans contained in the KnorpelPlus were enzymatically extracted from fish cartilage to a size in the range between 1500 to 2500 Da. This enzyme portioning of glycosaminoglycans is very important for later uptake of these agents into the bloodstream and therefore for their function in the joint. Only molecules of the right size are able to pass the mucous membrane of the intestine at a high concentration and are not digested or denatured.

\section{Acknowledgements}

This work was supported by the Internal Grant Agency of 
the Ministry of Health of the Czech Republic grant (IGA) No. 8122-3/2004, 210060, the Grant Agency of AS CR grants No. 1ET400110403 and IAA500390702, the Grant Agency of the Charles University in Prague grants No.
46/2005, 121/2005 and the Ministry of Education, Youth and Sport of the Czech Republic grants NPV II 2B06130 and MSM 6198959216.

\section{References}

ALTMAN R, MOSKOWITZ R: Hyaluronate sodium injections for osteoarthritis: the truth. Arch Intern Med 162: 24982499, 2002.

AMLER E, JASINSKA R, DRAHOTA Z, ZBOROWSKI J: Membrane lateral pressure as a modulator of glycerol-3phosphate dehydrogenase activity. FEBS Lett 271: 165-168, 1990.

BASSLEER C, HENROTIN Y, FRANCHIMONT P: In vitro evaluation of drugs proposed as chondroprotective agents. Int J Tissue React 14: 231-241, 1992.

BRIEF AA, MAURER SG, DI CESARE PE: Use of glucosamine and chondroitin sulfate in the management of osteoarthritis. J Am Acad Orthop Surg 9: 71-78, 2001.

BUCKWALTER JA, MANKIN HJ: Articular cartilage: tissue design and chondrocyte-matrix interactions. Instr Course Lect 47: 477-486, 1998.

COMBS GF JR: Impact of selenium and cancer-prevention findings on the nutrition-health paradigm (in Hebrew). Nutr Cancer 40: 6-11, 2001.

DEBI R, ROBINSON D, AGAR G, HALPERIN N: GAG for osteoarthritis of the knee - a prospective study. Harefuah 138: 451-453, 2000.

ERGGELET C, MANDELBAUM B, LAHM A: Der Knorpelschaden als therapeutische Aufgabe - Klinische Grundlagen. Dtsch Z Sportmed 51: 48-54, 2000.

FARNDALE RW, BUTTLE DJ, BARRETT AJ: Improved quantitation and discrimination of sulphated GAG by use of dimethylmethylene blue. Biochim Biophys Acta 883: 173-177, 1986.

FELSON DT, ANDERSON JJ: Hyaluronate sodium injections for osteoarthritis: hope, hype, and hard truths. Arch Intern Med 162: 245-247, 2002.

GRIMBLE RF: Nutritional antioxidants and the modulation of inflammation: theory and practice. New Horiz 2: $175-$ $185,1994$.

HARMAND MF, VILAMITJANA J, MALOCHE E, DUPHIL R, DUCASSOU D: Effects of S-adenosylmethionine on human articular chondrocyte differentiation. An in vitro study. Am J Med 83-A: 48-54, 1987.

HOMANDBERG GA, HUI F, WEN C, KUETTNER KE, WILLIAMS JM: Hyaluronic acid suppresses fibronectin fragment mediated cartilage chondrolysis: I. In vitro. Osteoarthritis Cartilage 5: 309-319, 1997.

LOTZ M: Cytokines in cartilage injury and repair. Clin Orthop Relat Res 391 (Suppl): S108-S115, 2001.

MACLEAN CH, KNIGHT K, PAULUS H, BROOK RH, SHEKELLE PG: Costs attributable to osteoarthritis. J Rheumatol 25: 2213-2218, 1998.

MARTINEK V, IMHOFF AB: Therapie von Knorpelschäden. Dtsch Z Sportmed 54: 70-76, 2003.

MARTINEK V, LATTERMAN C, USAS A, ABRAMOWITCH S, WOO SL, FU FH, HUARD J: Enhancement of tendon-bone integration of anterior cruciate ligament grafts with bone morphogenetic protein-2 gene transfer: a histological and biomechanical study. J Bone Joint Surg Am 84: 1123-1131, 2002.

MCALINDON TE, LAVALLEY MP, FELSON DT: Efficacy of glucosamine and chondroitin for treatment of osteoarthritis. JAMA 284: 1241, 2000a.

MCALINDON TE, LAVALLEY MP, GULIN JP, FELSON DT: Glucosamine and chondroitin for treatment of osteoarthritis: a systematic quality assessment and meta-analysis. JAMA 283: 1469-1475, $2000 \mathrm{~b}$.

NOACK W, FISCHER M, FORSTER K, ROVATI LC, SETNIKAR I: Glucosamine sulfate in osteoarthritis of the knee. Osteoarthritis Cartilage 2: 51-59, 1994.

O'DRISCOLL SW: The healing and regeneration of articular cartilage. J Bone Joint Surg 80-A: 1795-1812, 1998. 
PAVELKA K, GATTEROVÁ J, OLEJÁROVÁ M, MACHÁČEK S, GIACOVELLI G, ROVATI LC: Glucosamine sulfate use and delay of progression of knee osteoarthritis: a 3-year, randomized, placebo-controlled, doubleblind study. Arch Intern Med 162: 2113-2123, 2002.

PENG A, YANG CL: Examination of the roles of selenium in the Kaschin-Beck disease. Cartilage cell test and model studies. Biol Trace Elem Res 28: 1-9, 1991.

PENG A, YANG CL RUI H, LI H: Study on the pathogenic factors of Kashin-Beck disease. J Toxicol Environ Health 35: 79-90, 1992.

PIPERNO M, REBOUL P, HELLIO LE GRAVERAND MP, PESCHARD MJ, ANNEFELD M, RICHARD M, VIGNON E: Glucosamine sulfate modulates dysregulated activities of human osteoarthritic chondrocytes in vitro. Osteoarthritis Cartilage 8: 207-212, 2000.

PIPITONE VR: Chondroprotection with chondroitin sulfate. Drugs Exp Clin Res 17: 3-7, 1991.

RAYNAULD JP, TORRANCE GW, BAND PA, GOLDSMITH CH, TUGWELL P, WALKER V, SCHULTZ M, BELLAMY N: A prospective, randomized, pragmatic, health outcomes trial evaluating the incorporation of hylan G-F 20 into the treatment paradigm for patients with knee osteoarthritis (Part 1 of 2): clinical results. Osteoarthritis Cartilage 10: 506-517, 2002.

REGINSTER JY, DEROISY R, ROVATI LC, LEE RL, LEJEUNE E, BRUYERE O, GIACOVELLI G, HENROTIN Y, DACRE JE, GOSSETT C: Long-term effects of glucosamine sulphate on osteoarthritis progression: a randomised, placebo-controlled clinical trial. Lancet 357: 251-256, 2001.

RONCA F, PALMIERI L, PANICUCCI P, RONCA G: Anti-inflammatory activity of chondroitin sulfate. Osteoarthritis Cartilage 6 (Suppl): 14-21, 1998.

SETNIKAR I, PACINI MA, REVEL L: Antiarthritic effects of glucosamine sulfate studied in animal models. Arzneimittelforschung 41: 542-545, 1991.

SHARKEY PF, HOZACK WJ, ROTHMAN RH, SHASTRI S, JACOBY SM: Why are total knee arthroplasties failing today? Clin Orthop 404: 7-13, 2002.

STEINMEYER J: Die medikamentöse Therapie der Arthrose. Sport Orthop Traumatol 16: 19-25, 2000.

STEINMEYER J: Drug therapy of arthrosis. Orthopädie 30: 856-865, 2001.

TOWHEED TE: Published meta-analyses of pharmacological therapies for osteoarthritis. Osteoarthritis Cartilage 10: 836-837, 2002.

UEBELHART D, THONAR EJ, ZHANG J, WILLIAMS JM: Protective effect of exogenous chondroitin 4,6-sulfate in the acute degradation of articular cartilage in the rabbit. Osteoarthritis Cartilage 6 (Suppl): 6-13, 1998.

VIDAL Y, PLANA RR, BIZZARRI D, ROVATI AL: Articular cartilage pharmacology. In vitro studies on glucosamine and non-steroidal anti-inflammatory drugs. Pharmacol Res Commun 10: 557-569, 1978.

VIVIEN D, GALERA P, LOYAU G, PUJOL JP: N-acetyl-4-hydroxyproline (Oxaxeprol) and collagen synthesis in cultured synovial cells and articular cartilage. Osteoarthritis Cartilage 1: 40-45, 1993.

\section{Corresponding author}

M. Handl, Orthopedic Clinic, University Hospital Motol, V Úvalu 84, 15018 Prague 5, Czech Republic. Fax: 420224 432 820. E-mail: milan.handl@lfmotol.cuni.cz 